\title{
Diboson and multiboson results with CMS
}

\author{
Nate Woods*广 \\ University of Wisconsin-Madison (US) \\ E-mail: nwoods@hep.wisc.edu
}

\begin{abstract}
We summarize the results of recent studies of diboson and multiboson production performed on proton-proton collision data from the CMS detector at the LHC. Results discussed include ZZ, $\mathrm{W}^{ \pm} \mathrm{Z}$, and $\mathrm{W}^{+} \mathrm{W}^{-}$inclusive cross section measurements at $\sqrt{s}=13 \mathrm{TeV}$, as well as measurements of the $\mathrm{Z} \gamma \gamma, \mathrm{W}^{ \pm} \gamma \gamma, \gamma \gamma \rightarrow \mathrm{W}^{+} \mathrm{W}^{-}$, and electroweak $\mathrm{Z} \gamma+2 \mathrm{jets}$ and $\mathrm{W}^{ \pm} \gamma+2 \mathrm{jets}$ cross sections at $\sqrt{s}=8 \mathrm{TeV}$. Measurements done at $\sqrt{s}=8 \mathrm{TeV}$ are used to place limits on anomalous quartic gauge boson couplings.
\end{abstract}

Fourth Annual Large Hadron Collider Physics

13-18 June 2016

Lund, Sweden

* Speaker.

${ }^{\dagger}$ On behalf of the CMS collaboration 


\section{Introduction}

Diboson (VV, where $\mathrm{V}$ may indicate any of the electroweak gauge bosons, $\mathrm{Z}, \mathrm{W}^{ \pm}$, and $\gamma$ ) and triboson (VVV) production in proton-proton collisions is an important probe of standard model (SM) gauge boson interactions. The production cross sections for these processes are sensitive to deviations from the SM, and multi-V kinematic distributions provide a natural search channel for anomalous trilinear and quartic gauge couplings (aTGCs and aQGCs). Several multiboson processes are backgrounds to important Higgs boson discovery channels, and must be well understood to allow precision Higgs measuremets. Leptonic $\mathrm{W}^{ \pm}$and $\mathrm{Z}$ boson decays provide clean experimental signatures with few backgrounds, making multi- $\mathrm{V}$ measurement feasible despite their generally low cross sections.

We summarize recently released multiboson studies from the CMS experiment [1] at the CERN LHC. We first report early CMS diboson measurements performed on data collected in 2015 with center-of-mass energy $13 \mathrm{TeV}$ and an integrated luminosity of $\leq 2.6 \mathrm{fb}^{-1}$. This includes measurements of the $\mathrm{ZZ} \mathrm{[4],} \mathrm{W}^{ \pm} \mathrm{Z}$ [3], and $\mathrm{W}^{+} \mathrm{W}^{-}$[2] cross sections and a measurement of the $\mathrm{Z} \rightarrow 2 \ell 2 \ell^{\prime}$ branching fraction [4], where $\ell$ and $\ell^{\prime}$ indicate electrons or muons. All cross section measurements are performed in fully leptonic decay channels where $\mathrm{Z} \rightarrow \ell^{+} \ell^{-}$and $\mathrm{W}^{ \pm} \rightarrow \ell^{ \pm} v$. We then report analyses performed on data collected in 2012 with proton-proton center-of-mass energy $8 \mathrm{TeV}$ and an integrated luminosity of $\leq 19.7 \mathrm{fb}^{-1}$. This includes searches for electroweak $\mathrm{Z} \gamma$ [5] and $\mathrm{W}^{ \pm} \gamma[6]$ production in association with two jets, a search for exclusive or quasi-exclusive $\gamma \gamma \rightarrow \mathrm{W}^{+} \mathrm{W}^{-}$production [8], a search for $\mathrm{W}^{ \pm} \gamma \gamma$ triboson production [7], and an observation of $\mathrm{Z} \gamma \gamma$ triboson production [7]. All measurements on data with $\sqrt{s}=8 \mathrm{TeV}$ are used to place limits on dimension-8 anomalous gauge coupling operators.

\section{2. $\mathbf{W}^{+} \mathbf{W}^{-}$production}

The inclusive $\mathrm{W}^{+} \mathrm{W}^{-}$cross section was measured using $2.3 \mathrm{fb}^{-1}$ of data at $\sqrt{s}=13 \mathrm{TeV}$ in the $\mathrm{e}^{ \pm} \mu^{\mp} v v$ decay channel [2]. The $\mathrm{e}^{+} \mathrm{e}^{-} v v$ and $\mu^{+} \mu^{-} v v$ decay channels were neglected due to high backgrounds from Drell-Yan processes. Neutrinos escape the detector, leading to an imbalance in the transverse momentum sum of the particles detected in the event. The missing transverse momentum $\vec{p}_{T}^{m i s s}$ is the negative vector sum of the four-momenta of all observed particles in the event projected onto the plane parallel to the LHC beams. Its magnitude, the missing transverse energy $E_{T}^{\text {miss }}$, was required to be greater than $20 \mathrm{GeV}$. To suppress background events in which mismeasurement of the momentum of a lepton produces spurious $E_{T}^{\text {miss }}$, the projection of the missing transverse momentum onto the transverse momentum of the closest lepton, $\vec{p}_{T}^{\text {miss }} \cdot \vec{p}_{T}^{\ell}$, was required to be greater than $20 \mathrm{GeV}$. To suppress large backgrounds from top quark pair production, selected events were required to have no more than one jet with $p_{T}>30 \mathrm{GeV}$ and no jets with $p_{T}>20 \mathrm{GeV}$ identified as b-quark jets.

Distributions of $E_{T}^{\text {miss }}$ and the leading lepton $p_{T}$ for events selected in the 0 -jet category are shown in Fig. 1. The total $\mathrm{W}^{+} \mathrm{W}^{-}$cross section is calculated with the expression

$$
\sigma_{\mathrm{W}^{+} \mathrm{W}^{-}} \equiv \frac{N_{\mathrm{data}}-N_{\mathrm{bkg}}}{\mathscr{L}_{\mathrm{int}} \cdot \varepsilon \cdot(3 \cdot \mathscr{B}(\mathrm{W} \rightarrow \ell \boldsymbol{v}))^{2}}
$$


where $N_{\mathrm{data}}$ and $N_{\mathrm{bkg}}$ are the number of measured events in data and the number of expected background events, $\mathscr{L}_{\text {int }}$ is the integrated luminosity of the sample, $\varepsilon$ is the simulated signal efficiency, and $\mathscr{B}(\mathrm{W} \rightarrow \ell v)$ is the branching fraction of the $\mathrm{W}$ boson to each lepton family. It is found to be

$$
\sigma_{\mathrm{W}^{+} \mathrm{W}^{-}}=115.2 \pm 5.8(\text { stat }) \pm 5.7 \text { (syst) } \pm 6.4 \text { (theo) } \pm 3.6 \text { (lumi) } \mathrm{pb} .
$$

This can be compared to the theoretical value of $120.3 \pm 3.6 \mathrm{pb}$, calculated at next-to-next-toleading order (NNLO) in QCD.
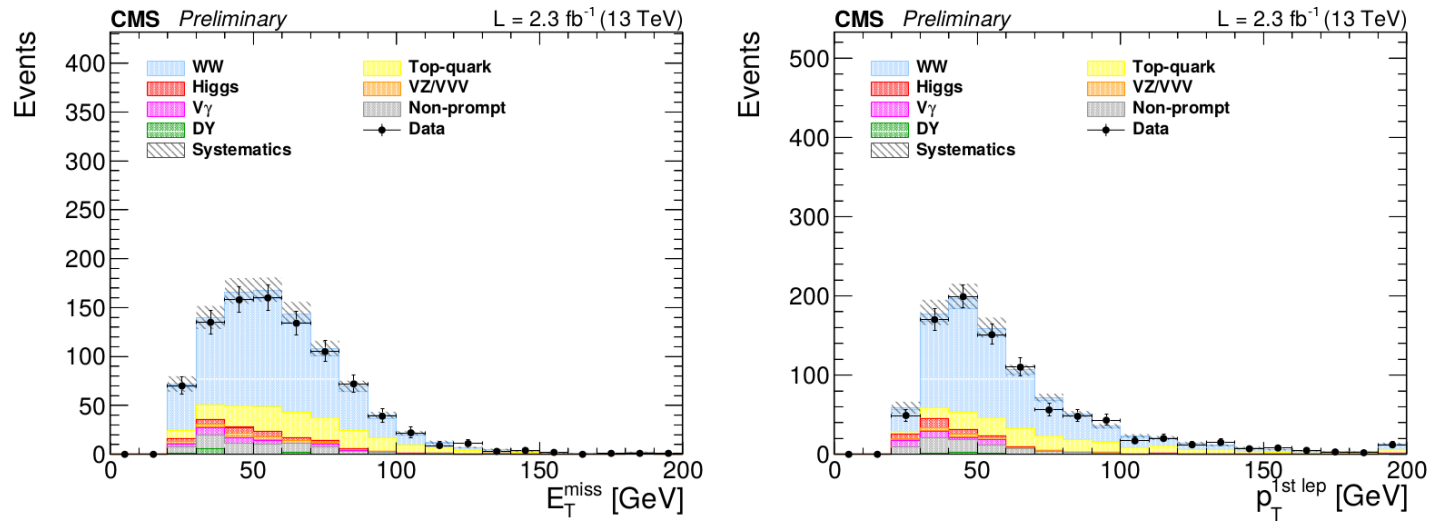

Figure 1: Distributions of (left) missing transverse energy and (right) the transverse momentum of the leading lepton in $\mathrm{W}^{+} \mathrm{W}^{-}$cross section measurement signal events. Black points represent measured data; shaded histograms represent expected yields, and the hatching represents the systematic uncertainty on the yield in each bin. The last bin is overflow.

\section{3. $\mathbf{W}^{ \pm} \mathbf{Z}$ production}

The inclusive $\mathrm{W}^{ \pm} \mathrm{Z}$ cross section was measured in the $\ell^{+} \ell^{-} \ell^{\prime \pm} v$ channel using $2.3 \mathrm{fb}^{-1}$ of data at $\sqrt{s}=13 \mathrm{TeV}$ [3]. The $\mathrm{Z}$ boson candidate was required to have invariant mass in the range $76<$ $m_{\ell^{+} \ell^{-}}<106 \mathrm{GeV}$, and each event was required to have $E_{T}^{\text {miss }}>30 \mathrm{GeV}$. To remove backgrounds from other diboson processes and t-quark pair production, events with a fourth lepton, or with a b-quark tagged jet with transverse momentum $p_{T}>30 \mathrm{GeV}$ and pseudorapidity $|\eta|<2.4$, were removed.

Background yields were estimated with a lepton fake-rate method, in which the probability for a jet to be misidentified as a prompt lepton was measured using a dijet control sample. This probability was applied to three-lepton control samples in which one, two, or all three leptons failed the full signal selection criteria. The uncertainty on the background yields leads to the largest systematic uncertainty on the total cross section, around 6\%.

The three-lepton invariant mass is shown in Fig. 2 (left). The total cross section $\sigma_{\mathrm{WZ}}$ is found for events in which the $\mathrm{Z}$ boson mass is in the range $60-120 \mathrm{GeV}$, with the difference in mass window accounted for by an acceptance correction found in simulated events. The measured value,

$$
\sigma_{\mathrm{WZ}}=40.9 \pm 3.4(\text { stat })_{-3.3}^{+3.1}(\text { syst }) \pm 0.4(\text { theo }) \pm 1.3(\text { lumi }) \mathrm{pb},
$$

can be compared to $42.6_{-0.8}^{+1.6} \mathrm{pb}$ calculated at next-to-leading order (NLO) in QCD with MCFM [9] or to $50.0_{-1.0}^{+1.1} \mathrm{pb}$ calculated at NNLO in QCD with MATRIX [10]. Figure 2 (right) shows the total 
cross section as a function of $\sqrt{s}$ from several measurements performed at LHC, compared to the NLO prediction.
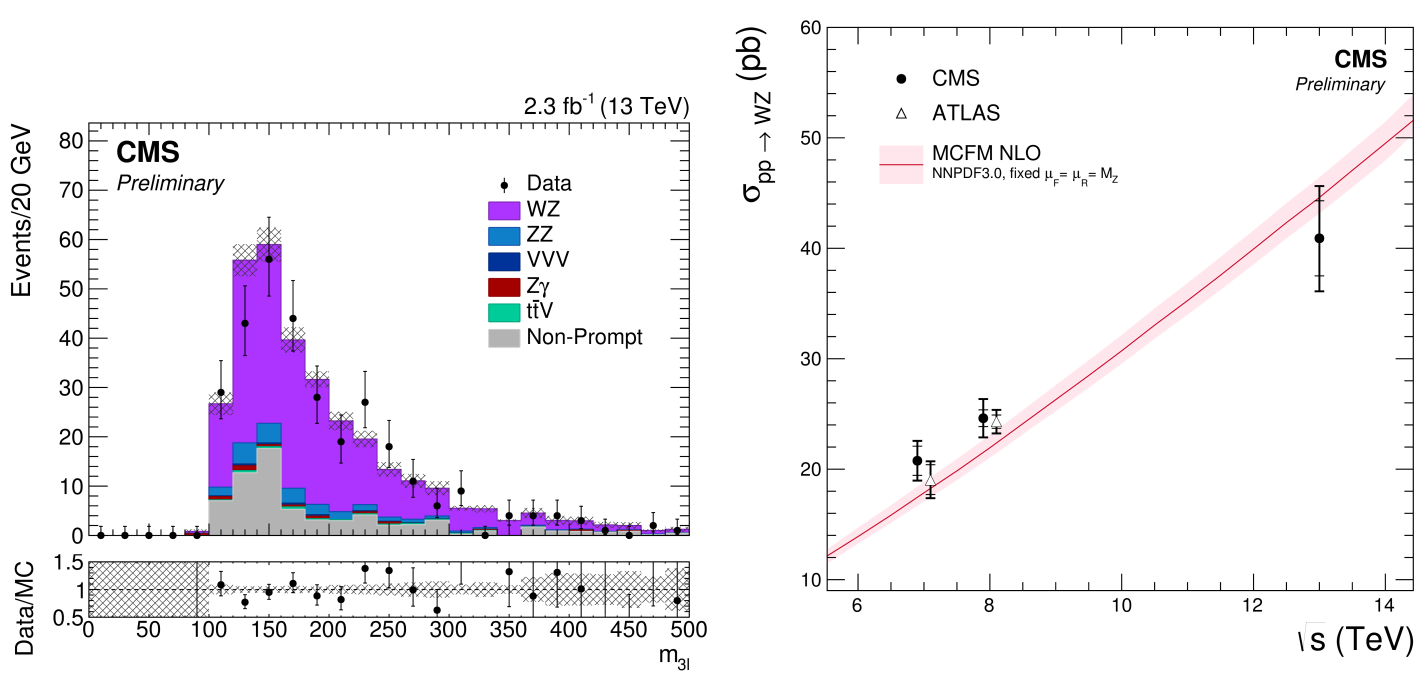

Figure 2: (left) Distribution of the three-lepton invariant mass in the $\mathrm{W}^{ \pm} \mathrm{Z}$ cross section measurement. Black points represent measured data, shaded histograms represent expected yields, and the hatching represents the systematic uncertainty on the yield in each bin. (right) Measured values of the $\mathrm{W}^{ \pm} \mathrm{Z}$ total cross section from the CMS (circles) and ATLAS (triangles) collaborations, and the corresponding NLO theory prediction (solid line) as a function of proton-proton center-of-mass energy.

\section{4. $\mathrm{ZZ}$ production}

The four-lepton spectrum, which includes $\mathrm{ZZ}, \mathrm{H} \rightarrow \mathrm{ZZ} \mathrm{Z}^{*}, \mathrm{Z} \gamma^{*}, \gamma^{*} \gamma^{*}$, and $\mathrm{Z} \rightarrow \ell \ell \gamma^{*}$ production, was found in the $\ell^{+} \ell^{-} \ell^{\prime+} \ell^{\prime-}$ channel using $2.6 \mathrm{fb}^{-1}$ of data at $\sqrt{s}=13 \mathrm{TeV}$ [4]. Each event was required to have two $\mathrm{Z} / \gamma^{*}$ candidates made of opposite-sign, same-flavor leptons, one with $40<m_{\ell \ell}<120 \mathrm{GeV}$, the other with $4<m_{\ell^{\prime} \ell^{\prime}}<120 \mathrm{GeV}$.

The full four-lepton mass spectrum and the $\mathrm{Z}$ boson mass spectrum are shown in Fig. 3, in which contributions from resonant $\mathrm{Z}$ and Higgs boson production and nonresonant $\mathrm{ZZ}$ production can be seen. The total $\mathrm{ZZ}$ cross section and the $\mathrm{Z} \rightarrow 4 \ell$ branching fraction were measured in subsets of this phase space.

The $\mathrm{Z} \rightarrow 4 \ell$ branching fraction was measured in events with $80<m_{4 \ell}<100 \mathrm{GeV}$. It is calculated with the expression

$$
\mathscr{B}(\mathrm{Z} \rightarrow 4 \ell)=\mathscr{B}(\mathrm{Z} \rightarrow 2 \ell) \times \frac{\sigma_{\mathrm{Z} \rightarrow 4 \ell}}{\sigma_{\mathrm{Z} \rightarrow 2 \ell} \mathscr{C}},
$$

where $\mathscr{B}(\mathrm{Z} \rightarrow 2 \ell)$ is the $\mathrm{Z}$ boson branching fraction to two electrons or two muons, $\sigma_{\mathrm{Z} \rightarrow 4 \ell}$ is the $\mathrm{Z} \rightarrow 4 \ell$ fiducial cross section measured in the window $80<m_{4 \ell}<100 \mathrm{GeV}, \sigma_{\mathrm{Z} \rightarrow 2 \ell}$ is the $\mathrm{Z} \rightarrow 2 \ell$ fiducial cross section in the mass window $60<m_{\ell \ell}<120 \mathrm{GeV}$ calculated with FEwZ [13], and $\mathscr{C}$ is a factor to correct for the acceptance difference between the two fiducial definitions and the small contribution from nonresonant $\gamma^{*} \gamma^{*}$ production. The branching fraction was found to be

$$
\mathscr{B}(\mathrm{Z} \rightarrow 4 \ell)=4.9_{-0.7}^{+0.8}(\text { stat })_{-0.2}^{+0.3}(\text { syst })_{-0.2}^{+0.3}(\text { theo }) \pm 0.1(\text { lumi }) \times 10^{-6},
$$



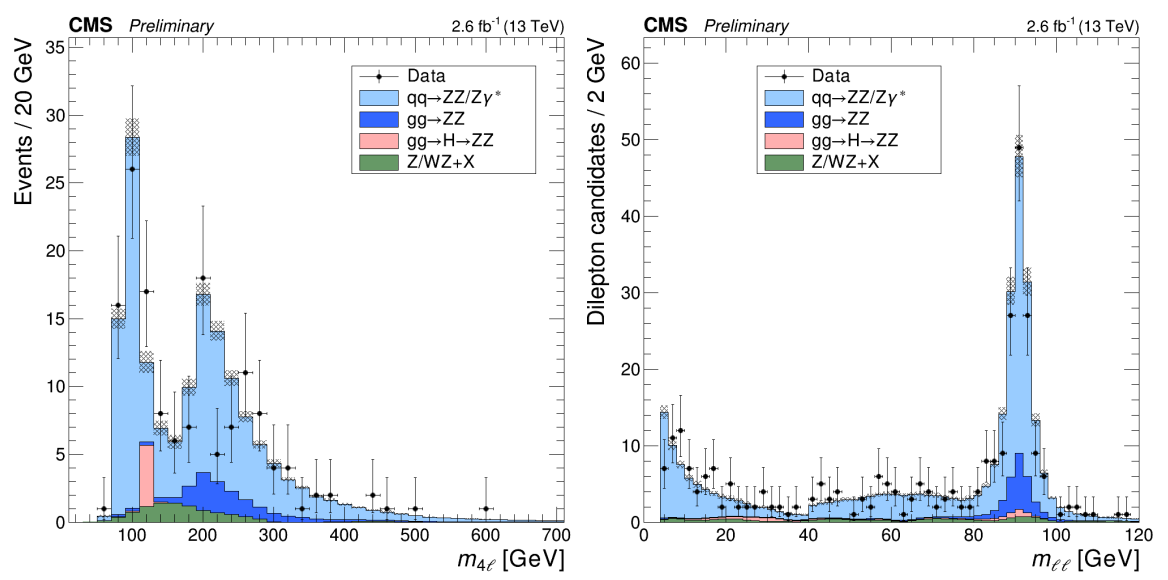

Figure 3: Distributions of (left) the four-lepton invariant mass and (right) the mass of all dilepton candidates in four-lepton events. Black points represent measured data; shaded histograms represent expected yields, and the hatching represents the systematic uncertainty on the yield in each bin.

which is consistent with the theoretical prediction of $4.6 \times 10^{-6}$, found with MCFM or MADGRAPH5_AMC@NLO.

The $\mathrm{ZZ}$ cross section was measured with both $\mathrm{Z}$ bosons required to be in the mass range $60<m_{\ell \ell}, m_{\ell^{\prime} \ell^{\prime}}<120 \mathrm{GeV}$. The four-lepton mass spectrum in this phase space is shown in Fig. 4 (left). The total inclusive cross section is found to be

$$
\sigma_{\mathrm{ZZ}}=14.6_{-1.8}^{+1.9}(\text { stat })_{-0.3}^{+0.5}(\text { syst }) \pm 0.2(\text { theo }) \pm 0.4(\text { lumi }) \mathrm{pb} .
$$

This may be compared to $15.0_{-0.6}^{+0.8} \mathrm{pb}$ calculated at next-to-leading order (NLO) in QCD with MCFM [9] or to $16.2_{-0.4}^{+0.6} \mathrm{pb}$ calculated at NNLO in QCD with MATRIX [12, 11] Figure 4 (right) shows the total cross section as a function of $\sqrt{s}$ from several measurements performed at LHC, compared to the predictions.

\section{Electroweak $\mathbf{Z} \gamma+2$ jets and $\mathbf{W}^{ \pm} \gamma+2$ jets}

Vector boson scattering (VBS) processes occur at $\mathscr{O}\left(\alpha_{E W K}^{5} \alpha_{s}^{0}\right)$, which allows them to be used as a probe of electroweak couplings independent of QCD effects. Though VBS cross sections are small, their distinctive topology, with a pair of high-rapidity jets, allows them to be reliably tagged and separated from backgrounds. Searches for electroweak production of $\mathrm{Z} \gamma$ and $\mathrm{W}^{ \pm} \gamma$ in association with two jets were performed on $19.7 \mathrm{fb}^{-1}$ of data at $\sqrt{s}=8 \mathrm{TeV}$.

The electroweak $Z \gamma+2$ jets search [5], performed in the $\mathrm{e}^{+} \mathrm{e}^{-} \gamma j j$ and $\mu^{+} \mu^{-} \gamma j j$ channels, required the $\mathrm{Z}$ boson to be in the mass range $70<m_{\ell \ell}<110 \mathrm{GeV}$. The VBS jets were taken to be the two highest $p_{T}$ jets in the event, with $p_{T}>30 \mathrm{GeV}$ and $|\eta|<4.7$. The dijet invariant mass in the $\mathrm{Z} \rightarrow \mu^{+} \mu^{-}$channel is shown in Fig. 5 (left) with these selections. The signal sample is defined with further cuts to select for the VBS topology. Selections are placed on the dijet invariant mass and pseudorapidity separation, $m_{j j}>400 \mathrm{GeV}$ and $\Delta \eta_{j j}>1.6$. The azimuthal angular separation between the $\ell \ell \gamma$ system and the dijet system was required to be $\Delta \phi_{Z \gamma, j j}>2.0$, and a selection was placed on the Zeppenfeld variable, $\left|y_{Z \gamma}-\frac{1}{2}\left(y_{j_{1}}+y_{j_{2}}\right)\right|<1$, where $y_{Z \gamma}, y_{j_{1}}$, and $y_{j_{2}}$ are the 

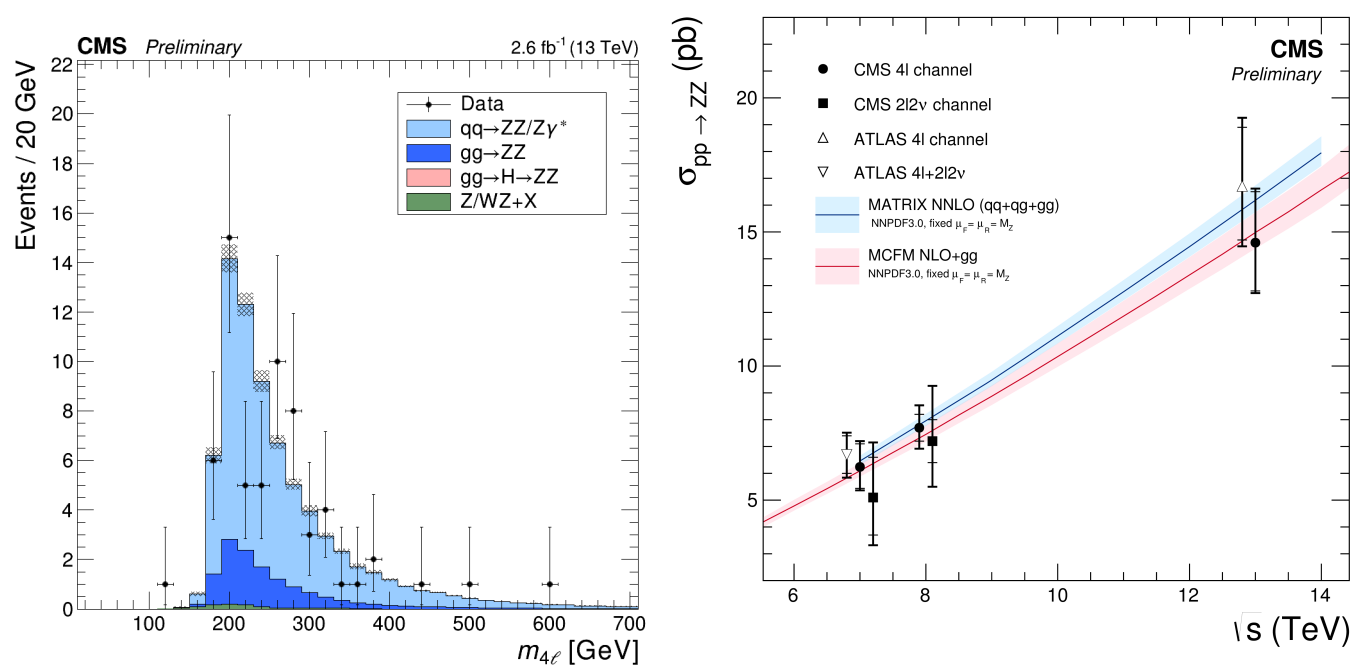

Figure 4: (left) Distribution of the four-lepton invariant mass of events with $60<m_{\ell \ell}, m_{\ell^{\prime} \ell^{\prime}}<120 \mathrm{GeV}$, used to measure the $\mathrm{ZZ}$ cross section. Black points represent measured data; shaded histograms represent expected yields, and the hatching represents the systematic uncertainty on the yield in each bin. (right) Measured values of the total ZZ cross section from the CMS (circles) and ATLAS (triangles) experiments, along with the corresponding NLO and NNLO predictions (solid curves), as a function of the proton-proton center-of-mass energy.

rapidities of the $\mathrm{Z} \gamma$ system and the two jets. The invariant mass of the $\mathrm{Z} \gamma$ system after the signal selections is shown in Fig. 5 (right), along with an example aQGC signal shape.

Evidence of electroweak $Z \gamma+2$ jets production was found with a significance of $3.0 \sigma$ above the background-only expectation ( $2.1 \sigma$ expected). The VBS Z $\gamma$ cross section was found to be

$$
\sigma_{\mathrm{Z} \gamma}^{\text {ewk }}=1.86_{-0.75}^{+0.89}(\text { stat })_{-0.27}^{+0.41} \text { (syst) } \pm 0.05 \text { (lumi) fb, }
$$

which is compatible with the leading-order (LO) MADGRAPH expectation of $1.26 \pm 0.11$ (scale) \pm 0.05 (PDF) fb.

The electroweak $\mathrm{W}^{ \pm} \gamma+2$ jets search [6], performed in the $\mathrm{e}^{ \pm} \gamma j j+E_{T}^{\text {miss }}$ and $\mu^{ \pm} \gamma j j+E_{T}^{\text {miss }}$ channels, followed a similar strategy, but with specific selections tightened to remove larger backgrounds. Each event was required to have $E_{T}^{\text {miss }}>35 \mathrm{GeV}$. The dijet invariant mass for $\mathrm{e}^{ \pm} \gamma j j+$ $E_{T}^{\text {miss }}$ events passing these criteria is shown in Fig. 6 (left). Events in the signal sample were further required to have $m_{j j}>700 \mathrm{GeV}, \Delta \eta_{j j}>1.6, \Delta \phi_{\mathrm{W} \gamma, j j}>2.6$, and $\left|y_{\mathrm{W} \gamma}-\frac{1}{2}\left(y_{j_{1}}+y_{j_{2}}\right)\right|<0.6$. The transverse momentum of $\mathrm{W}$ bosons in the VBS signal region is shown in Fig. 6 (right), along with an example aQGC signal shape.

An excess consistent with electroweak $\mathrm{W}^{ \pm} \gamma+2 \mathrm{jets}$ production was found at the level of $2.7 \sigma$ over background ( $1.5 \sigma$ expected). The VBS $\mathrm{W}^{ \pm} \gamma$ cross section was found to be

$$
\sigma_{\mathrm{W}^{ \pm} \gamma}^{\text {ewk }}=10.8 \pm 4.1(\text { stat }) \pm 3.4(\text { syst }) \pm 0.3(\text { lumi }) \mathrm{fb},
$$

which is compatible with the NLO MADGRAPH expectation of $6.1 \pm 1.2$ (scale) \pm 0.2 (PDF) fb. 

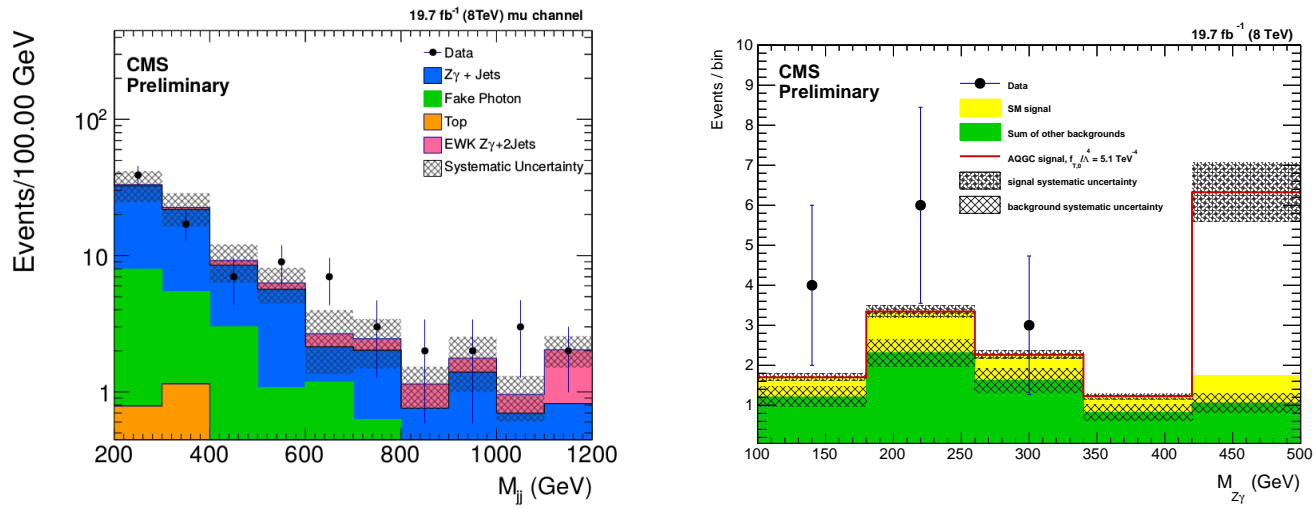

Figure 5: Distributions of (left) the dijet invariant mass in $\mu^{+} \mu^{-} \gamma j j$ events before VBS selections and (right) the invariant mass of the $\ell^{+} \ell^{-} \gamma$ system after VBS selections. Black points represent measured data; shaded histograms represent expected yields, and the hatching represents the systematic uncertainty on the yield in each bin. The unfilled red histogram represents an example signal shape with aQGC.
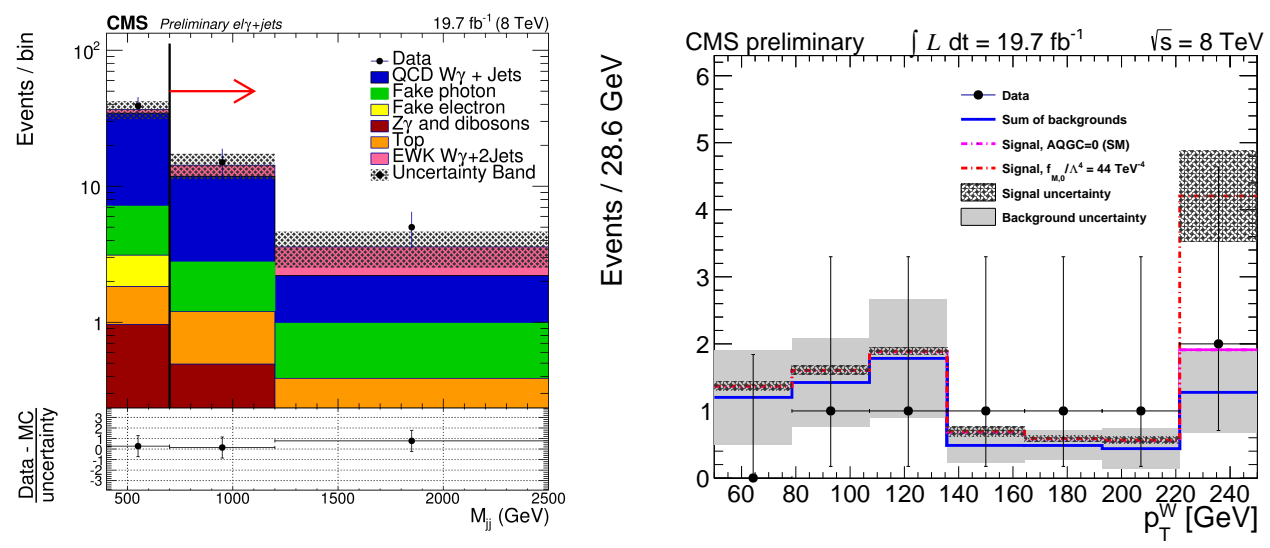

Figure 6: Distributions of (left) the dijet invariant mass in $\mathrm{e}^{ \pm} \gamma j j+E_{T}^{\text {miss }}$ events before VBS selections and (right) the transverse momentum of the $\mathrm{W}^{ \pm}$boson after VBS selections. Black points represent measured data; shaded histograms represent expected yields, and the hatching represents the systematic uncertainty on the yield in each bin. The unfilled red histogram represents an example signal shape with aQGC.

\section{6. $\mathbf{Z} \gamma \gamma$ and $\mathbf{W}^{ \pm} \gamma \gamma$ production}

Searches for $\mathrm{Z} \gamma \gamma$ and $\mathrm{W}^{ \pm} \gamma \gamma$ production were performed using $19.4 \mathrm{fb}^{-1}$ of data at $\sqrt{s}=$ $8 \mathrm{TeV}$ [7]. The $\mathrm{Z} \gamma \gamma$ search was performed in the $\mathrm{e}^{+} \mathrm{e}^{-} \gamma \gamma$ and $\mu^{+} \mu^{-} \gamma \gamma$ channels, with the $\mathrm{Z}$ boson candidate required to satisfy $m_{\ell \ell}>40 \mathrm{GeV}$. The $\mathrm{W}^{ \pm} \gamma \gamma$ search was performed in the $\mu^{ \pm} \gamma \gamma+E_{T}^{\text {miss }}$ channel, with the transverse mass of the $\mathrm{W}^{ \pm}$boson, $m_{T}^{\mathrm{W}} \equiv \sqrt{2 E_{T}^{\text {miss }} p_{T}^{\ell^{\prime}}(1-\cos (\Delta \phi))}$, required to be greater than $40 \mathrm{GeV}$.

The largest backgrounds come from $\mathrm{V}+$ jets events in which one or two jets are misidentified as photons. The misidentification probability was found using a $Z+j e t s$ control sample, and was applied to template shapes for events with zero, one, and two fake photons to find the background yield in the signal sample. The jet to photon misidentification probability uncertainty is the primary systematic uncertainty on the measured cross sections, around $37 \%$ for $\sigma_{\mathrm{W} \gamma \gamma}$ and $14 \%$ for $\sigma_{\mathrm{Z} \gamma \gamma}$. 
The diphoton invariant mass for $Z \gamma \gamma$ events is shown in Fig. 7 (left) in the $\mu^{+} \mu^{-} \gamma \gamma$ channel. $\mathrm{Z} \gamma \gamma$ production was observed with a significance of $5.9 \sigma$ over the background expectation. The fiducial cross section was found to be

$$
\sigma_{\mathrm{Z} \gamma \gamma}^{\mathrm{fid}} \times \mathscr{B}\left(\mathrm{Z} \rightarrow \ell^{+} \ell^{-}\right)=12.7 \pm 1.4(\text { stat }) \pm 1.8(\text { syst }) \pm 0.3 \text { (lumi) fb }
$$

which is compatible with the NLO MADGRAPH prediction of $12.95 \pm 1.47 \mathrm{fb}^{-1}$.

The diphoton invariant mass for $\mathrm{W}^{ \pm} \gamma \gamma$ events is shown in Fig. 7 (right). An excess consistent with $\mathrm{W}^{ \pm} \gamma \gamma$ production was seen with a significance of $2.4 \sigma$ over the background expectation. The fiducial cross section was found to be

$$
\sigma_{\mathrm{W}^{ \pm} \gamma \gamma}^{\mathrm{fid}} \times \mathscr{B}\left(\mathrm{W}^{ \pm} \rightarrow \ell^{ \pm} v\right)=6.0 \pm 1.8 \text { (stat) } \pm 2.3 \text { (syst) } \pm 0.2 \text { (lumi) fb }
$$

which is compatible with the NLO MADGRAPH prediction of $4.76 \pm 0.53 \mathrm{fb}^{-1}$.
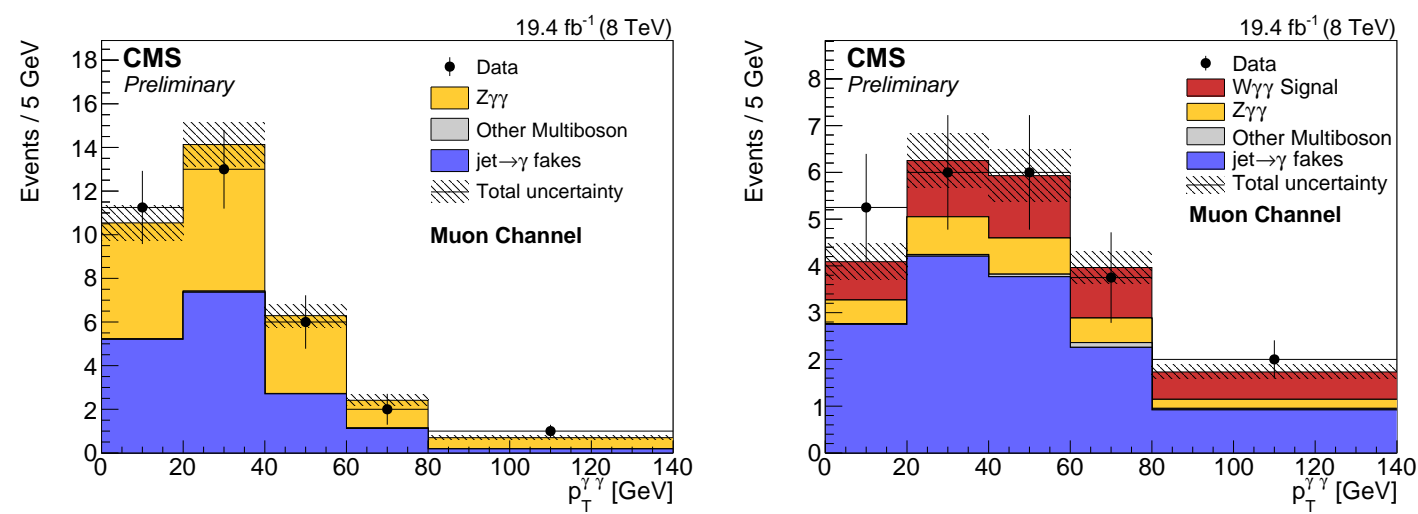

Figure 7: Distributions of diphoton invariant mass for (left) $Z \gamma \gamma$ events in the $\mu^{+} \mu^{-} \gamma \gamma$ decay channel and (right) $\mathrm{W}^{ \pm} \gamma \gamma$ events in the $\mu^{ \pm} \gamma \gamma+E_{T}^{\text {miss }}$ channel. Black points represent measured data, shaded histograms represent expected yields, and the hatching represents the total uncertainty on the yield in each bin.

\section{Exclusive and quasi-exclusive $\gamma \gamma \rightarrow \mathbf{W}^{+} \mathbf{W}^{-}$production}

The $\gamma \gamma \mathrm{W}^{+} \mathrm{W}^{-}$quartic coupling and $\gamma \mathrm{W}^{+} \mathrm{W}^{-}$trilienar coupling can be probed directly in $\gamma \gamma \rightarrow \mathrm{W}^{+} \mathrm{W}^{-}$production. This process occurs at the LHC when a pair of protons scatters through quasi-real photon interactions, $\mathrm{pp} \rightarrow \mathrm{p}^{(*)} \mathrm{W}^{+} \mathrm{W}^{-} \mathrm{p}^{(*)}$. The protons may scatter elastically ("exclusive") or scatter inelastically and dissociate ("quasi-exclusive"), but in either case they or their remnants fall outside the detector acceptance.

The search for exclusive $\gamma \gamma \rightarrow \mathrm{W}^{+} \mathrm{W}^{-}$production was performed using $19.7 \mathrm{fb}^{-1}$ of data at $\sqrt{s}=8 \mathrm{TeV}$ [8]. To remove large backgrounds arising from Drell-Yan and exclusive $\gamma \gamma \rightarrow \ell^{+} \ell^{-}$ production, the opposite-flavor decay channel $\mathrm{W}^{+} \mathrm{W}^{-} \rightarrow \mathrm{e}^{ \pm} \mu^{\mp} v v$ was used. The resulting oppositely charged electron and muon were required to originate from a common interaction vertex with no other associated charged tracks, to remove inclusive diboson events and other backgrounds which are generally associated with extra charged particles from underlying event activity or multiparton interactions. To remove events from exclusive $\gamma \gamma \rightarrow \tau^{+} \tau^{-}$interactions, the electron-muon system is required to have $p_{T}>30 \mathrm{GeV}$. 
The same-flavor decay channels were used as control samples to understand the efficiency of the signal sample, background yields, and poorly-modeled nonperturbative effects like proton dissociation. Exclusive $\gamma \gamma \rightarrow \ell^{+} \ell^{-}$events were selected by requiring the leptons to be coplanar, with $\left|1-\Delta \phi_{\ell \ell} / \pi\right|<0.01$., and by requiring their invariant mass to be inconsistent with a $\mathrm{Z}$ boson, with $m_{\ell \ell}<70 \mathrm{GeV}$ or $m_{\ell \ell}>106 \mathrm{GeV}$.

The invariant mass of the $\mathrm{e}^{ \pm} \mu \mp$ system is shown in Fig. 8 (left). The $p_{T}$ of the system is shown in Fig. 8 (right), along with example aTGC signal shapes. When combined with similar results performed on $7 \mathrm{TeV}$ data [14], evidence of $\gamma \gamma \rightarrow \mathrm{W}^{+} \mathrm{W}^{-}$production was seen with a significance of $3.4 \sigma$ over the background expectation ( $2.8 \sigma$ expected). The measured cross section was found to be $11.9_{-4.5}^{+5.6} \mathrm{fb}$, which can be compared to the MADGRAPH prediction of $6.9 \pm 0.6 \mathrm{fb}$.
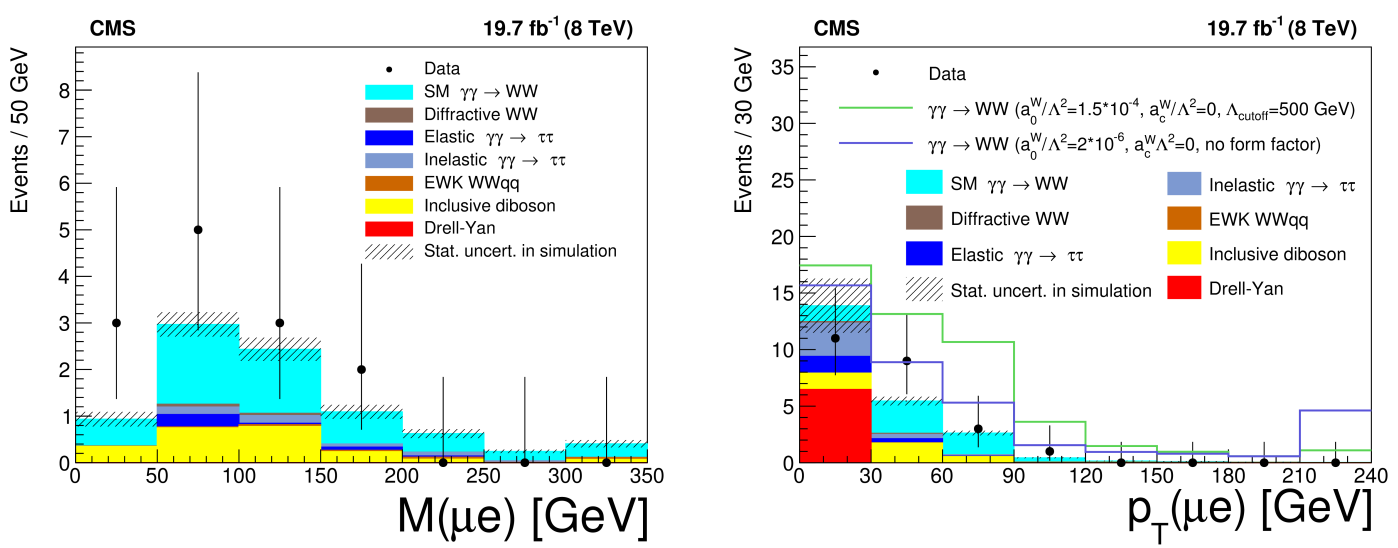

Figure 8: Distributions of (left) the invariant mass of the $\mathrm{e}^{ \pm} \mu \mp$ system and (right) the $p_{T}$ of the $\mathrm{e}^{ \pm} \mu \mp$ system for events used in the exclusive $\gamma \gamma \rightarrow \mathrm{W}^{+} \mathrm{W}^{-}$search. Black points represent measured data, shaded histograms represent expected yields, and the hatching represents the statistical uncertainty on the simulated yield in each bin. Hollow histograms show example anomalous gauge coupling signal shapes.

\section{Summary of cross sections and aQGC limits}

A summary of CMS diboson cross sections measurements, with comparisons to theoretical predictions, is shown in Fig. 9. Cross sections for electroweak, exclusive, and triboson processes measured by CMS at $\sqrt{s}=8 \mathrm{TeV}$ are summarized in Fig. 10. Experimental results are overall compatible with theory.

All measurements described above which used $\sqrt{s}=8 \mathrm{TeV}$ data were used to set limits on dimension-8 aQGC operators. A summary of such limits is shown in Fig. 11.

\section{References}

[1] CMS Collaboration, The CMS experiment at the CERN LHC, JINST 3, S08004 (2008).

[2] CMS Collaboration Measurement of the WW cross section in pp collisions at $\operatorname{sqrt}(\mathrm{s})=13 \mathrm{TeV}$, CMS-PAS-SMP-16-006, https://cds.cern.ch/record/2160868.

[3] CMS Collaboration, Measurement of the WZ production cross section in pp collisions at sqrt $(s)=13$ $\mathrm{TeV}$, CMS-PAS-SMP-16-002, https://cds.cern.ch/record/2144880. 


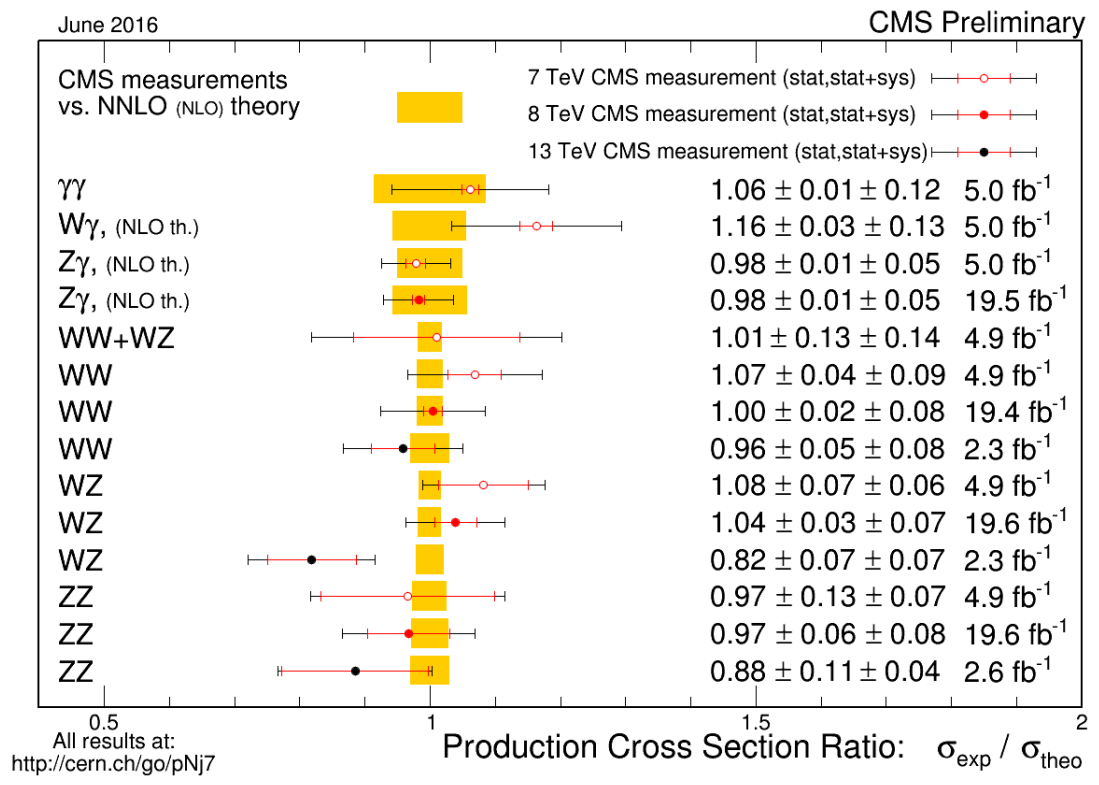

Figure 9: Summary of CMS diboson cross section measurements, compared to theoretical predictions.

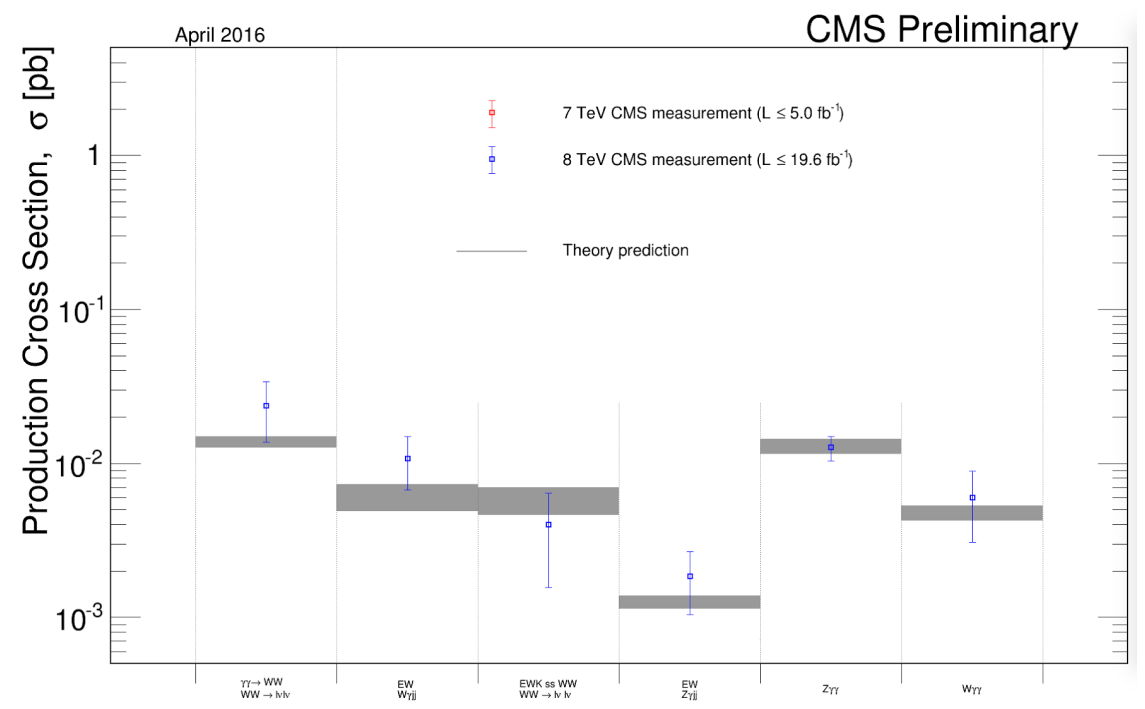

Figure 10: Summary of CMS triboson, electroweak, and exclusive production cross section measurements, compared to theoretical predictions.

[4] CMS Collaboration, Measurement of the ZZ production cross section and Z to lll'l' branching fraction in pp collisions at $\operatorname{sqrt}(s)=13 \mathrm{TeV}$, CMS-PAS-SMP-16-001, https://cds.cern.ch/record/2148595.

[5] CMS Collaboration, Evidence for the electroweak Zgamma production in association with two jets and a search for anomalous quartic gauge couplings in pp collisions at $\operatorname{sqrt}(\mathrm{s})=8 \mathrm{TeV}$, CMS-PAS-SMP-14-018, https://cds.cern.ch/record/2048148.

[6] CMS Collaboration, A search for electroweak-induced production of Wgamma with two jets and constraints on anomalous quartic gauge couplings in pp collisions at $\operatorname{sqrt}(\mathrm{s})=8 \mathrm{TeV}$, CMS-PAS-SMP-14-011, https://cds.cern.ch/record/2124432. 


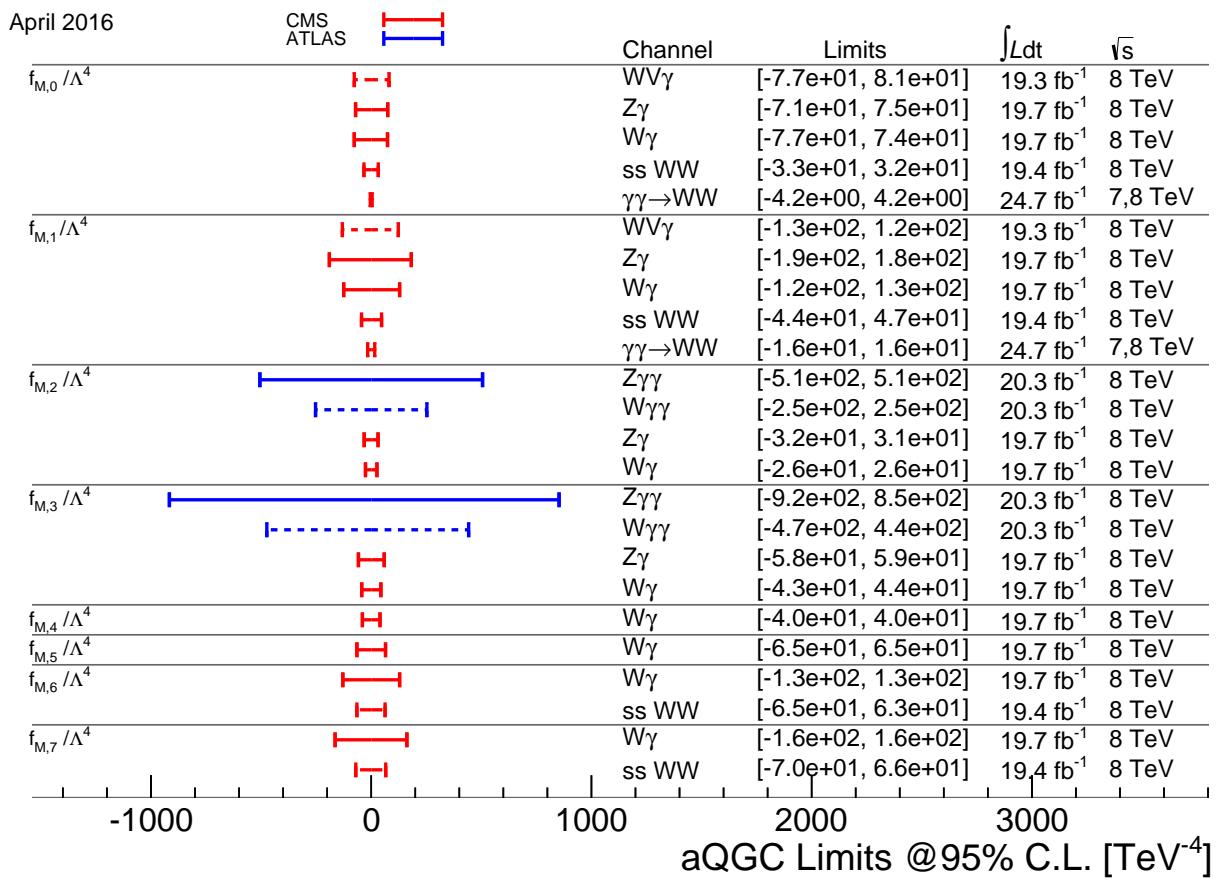

Figure 11: Summary of limits on dimension- 8 aQGC operators affecting both transverse and longitudinal modes.

[7] CMS Collaboration, Measurements of the pp to Wgammagamma and pp to Zgammagamma cross sections and limits on dimension-8 effective anomalous gauge couplings at sqrt $(s)=8 \mathrm{TeV}$, CMS-PAS-SMP-15-008, https://cds.cern.ch/record/2130360.

[8] CMS Collaboration, Evidence for exclusive gammagamma to WW production and constraints on anomalous quartic gauge couplings at sqrt(s) $=7$ and $8 \mathrm{TeV}$, submitted to JHEP, [hep-ex/1604.04464].

[9] J. Campbell and R. Ellis, MCFM for the Tevatron and the LHC, in proceedings of the 10th DESY Workshop on Elementary Particle Theory: Loose and Legs in Quantum Field Theory, Nucl. Phys. Proc. Suppl. 10 (2010) [hep-ph/1007.3492].

[10] M. Grazzini, S. Kallweit, D. Rathlev and M. Wiesemann, WZ production at hadron colliders in NNLO QCD, Phys. Lett. B 761 (2016) [hep-ph/1604.08576].

[11] M. Grazzini, S. Kallweit, and D. Rathlev, ZZ production at the LHC: fiducial cross sections and distributions in NNLO QCD, Phys. Lett. B 750 (2015) [hep-ph/1507. 06257].

[12] F. Cascioli et al., ZZ production at hadron colliders in NNLO QCD, Phys. Lett. B 735 (2014) [hep-ph/1405.2219].

[13] R. Gavin, Y. Li, F. Petriello, and S. Quackenbush, FEWZ 2.0: A code for hadronic Z production at next-to-leading order, Comput. Phys. Commun. 182 (2011) [hep-ph/1011.3540].

[14] CMS Collaboration, Study of exclusive two-photon production of WW in pp collisions at sqrt $(s)=7$ $\mathrm{TeV}$ and constraints on anomalous quartic gauge couplings, JHEP 07 (2013) [hep-ex/1305.5596]. 